\title{
EL PROGRAMA DE DOCTORADO EN PROBLEMAS ECONÓIMICO AGROINDUSTRIALES, UNIVERSIDAD AUTÓNOMA CHAPINGO (MÉXICO).
}

\author{
DOCTORAL PROGRAM IN ECONOMIC AGROINDUSTRIAL \\ PROBLEMS, AUTONOMOUS UNIVERSITY OF CHAPINGO (MEXICO).
}

Fernando Cervantes Escoto ${ }^{1}$

MÉXICO

Palabras Clave: Desarrollo agropecuario, industria, economía, universidad-estado.

Keywords: Agricultural development, industry, economy, universitystate.

\section{Resumen}

El programa de Doctorado en Problemas Económico Agroindustriales es un programa de posgrado que promueve compromisos con la sociedad rural y urbana, al fomentar el desarrollo personal del investigador, la creatividad académica y generar colectivamente conocimientos que ayuden a comprender y orientar el curso del desarrollo agropecuario del país, en un ámbito en donde hay cabida para todas las ideologías y las estructuras disciplinarias. Además, busca reafirmar los valores de la sociedad a través del enriquecimiento de los individuos que estudian en sus aulas. Así, profesores y alumnos buscan el logro de estos objetivos en forma libre y responsable, en un ambiente en donde priva la excelencia académica, el humanismo, la honradez, el trabajo creativo y colectivo y la civilidad de los participantes.

\section{Abstract}

This paper presents the doctoral program in Economic and Agroindustrial Problems, which promotes commitments with rural and urban society. This effort fosters personal development of researchers and their academic creativity. Also, this helps to create collective knowledge that allows understanding and orients the path of the agricultural development of Mexico, a place for all ideologies and disciplinary structures. In addition, it seeks to strenghthen social values through the enrichment of students. Thus, professors

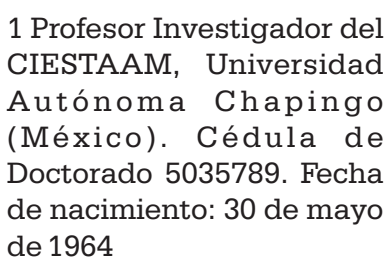
CIESTAAM, Universidad Autónoma Chapingo (México). Cédula de Doctorado 5035789. Fecha de nacimiento: 30 de mayo de 1964

Pre-aprobado Congreso. Aprobado ÁNFORA ago 05/09

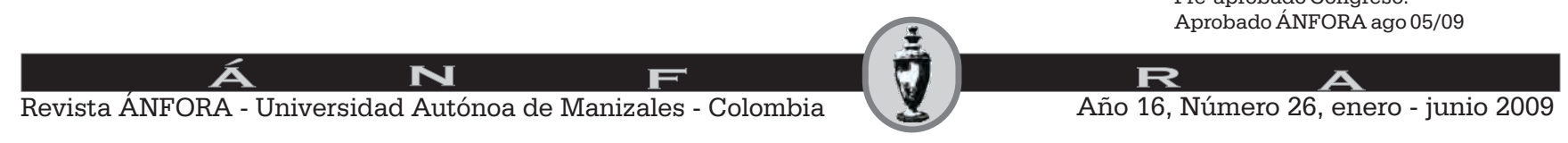


and students attempt to achieve these objectives in a free and responsible atmosphere, where academic excellence, humanism, honesty, civility, creative, and collective work of participants prevail.

\section{Introducción}

La problemática actual de México, que se combina con viejos problemas no superados del sector agropecuario, hace necesario revisar los esquemas teóricos y los enfoques que se han ido generando y poniendo en marcha ante la situación de crisis de la agricultura nacional y mundial, a fin de que la Universidad Autónoma Chapingo participe con nuevas opciones en la formación de recursos humanos del más alto nivel académico, capaces de analizar con rigor científico, en su causalidad y manifestaciones específicas esta nueva realidad, de manera que puedan proponer acciones concretas con la finalidad de contribuir a la solución de dicha problemática.

El CIESTAAM (Centro de Investigaciones Económicas, Sociales y Tecnológicas de la Agroindustria y la Agricultura Mundial), de la Universidad Autónoma Chapingo, desde 1990 ha desarrollado una gran experiencia en investigación, la cual está dirigida a aportar elementos que permitan que las iniciativas para la formulación de políticas orientadas al desarrollo del sector agropecuario incluyan además de las variables macroeconómicas, las condiciones microeconómicas específicas del campo. Dicha experiencia le ha permitido al Centro, como parte de la estructura universitaria, participar en un programa de posgrado, el cual está concebido con la idea de fortalecer y ampliar la capacidad de investigación mediante la formación de investigadores a nivel de Doctorado.

Se pretende mediante este programa de posgrado, ofrecer a los alumnos la oportunidad de la asesoría de investigadores experimentados, la infraestructura y el ambiente de un grupo de trabajo y una forma o estilo del quehacer científico. Todo lo cual ayudará a constituir una opción diferente para la formación de personal con un enfoque "tutorial".

Desde 1996, la Universidad Autónoma Chapingo (UACh) ofrece el Doctorado en Problemas Económico-Agroindustriales, con sede en el CIESTAAM. Centro respaldado por una sólida experiencia de investigación, difusión de sus resultados y vinculación con las instituciones nacionales del sector agropecuario, organizaciones de productores e instituciones internacionales y nacionales de investigación y educación, ha sido capaz de ofrecer las mejores condiciones para la formación de sus doctorandos y ha logrado, además de una elevada eficiencia terminal, que algunos de sus trabajos de tesis sean premiados en concursos nacionales, que sus estudiantes realicen estancias de estudio en universidades extranjeras y que sus profesores participen directamente en el diseño y ejecución o evaluación de la política agrícola que actualmente se aplica en el campo mexicano.

Así, el Doctorado ofrece a la sociedad mexicana investigadores de alto nivel científico y técnico, con capacidad ejecutiva y una visión amplia que les permite incidir en la actual problemática del campo. Las primeras generaciones de

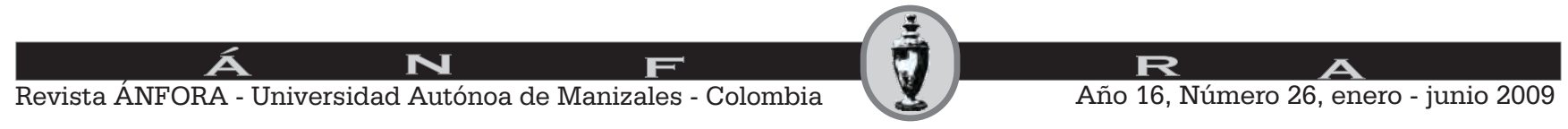


egresados se han insertado exitosamente en centros de investigación, instituciones educativas, organizaciones no gubernamentales y en labores de consultoría. De esa manera, el Doctorado en Problemas EconómicoAgroindustriales ha venido cumpliendo con los objetivos planteados en su origen (ver más adelante).

Para alcanzar los objetivos y ejercer un papel crecientemente protagónico ante la sociedad mexicana, la instancia coordinadora de este Doctorado ha mantenido una actitud autocrítica con respecto a su Plan de Estudios y a las actividades específicas de docencia e investigación, de tal forma que se han introducido diversas mejoras, tanto en la composición del Plan de Estudios como en el contenido y ejecución de cada una de las materias. De igual forma, se han afinado los procedimientos para dar seguimiento a la formación de cada estudiante, a fin de garantizar resultados de investigación de calidad, y se ha avanzado en la reglamentación y en la constitución de una base de recursos humanos, financieros y materiales que garantizan una excelencia académica y aseguran continuidad en el cumplimiento de los objetivos.

En alto grado, lo anterior ha sido influenciado por las recomendaciones que en evaluaciones precedentes ha hecho el CONACYT, las cuales se han concretado en la introducción de cursos básicos, en el establecimiento de prioridades para el ingreso de estudiantes y en la consolidación de su planta docente por medio del ingreso en el Sistema Nacional de Investigadores. No obstante lo anterior, el programa mantiene los rasgos esenciales que le dieron origen, a saber: una orientación centrada en la investigación, un carácter tutorial y una alta flexibilidad, sin perder por ello la rigurosidad y disciplina correspondientes.

Con ese antecedente, a continuación se exponen la justificación, viabilidad, objetivos y metas del Doctorado, previamente a la presentación del Plan de Estudios.

\section{JUSTIFICACIÓN DEL DOCTORADO}

La situación actual del país, caracterizada por una inserción de la economía en relaciones internacionales más abiertas, presenta grandes retos para el desarrollo del sector agropecuario, forestal y agroindustrial, a la vez que enfrenta importantes problemas que demandan solución, en la medida en que el fenómeno de la globalización y las estrategias de ajuste estructural adoptadas por el Estado mexicano introducen nuevas condiciones a los distintos grupos de productores y agentes participantes en las cadenas agroalimentarias.

Está a prueba la capacidad de todo tipo de productores para sostener sus actividades frente a una creciente competencia internacional, al mismo tiempo que se han redefinido las funciones del Estado hacia el sector rural y se transita hacia una nueva inserción de la agricultura en el desarrollo económico, originándose, en consecuencia, nuevas formas de integración agriculturaindustria y nuevos retos para que los productores y sus organizaciones asuman

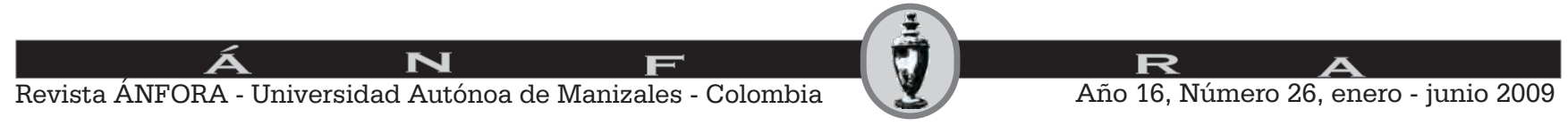


funciones y responsabilidades que el Estado ha dejado en manos de la operación libre de los mercados.

Con un nivel de mayor detalle, puede decirse que la agricultura mexicana se ha visto sometida desde 1982 a un profundo proceso de transformaciones que, junto con la crisis nacional, ha generado una nueva problemática en el sector agropecuario y forestal. Este es el resultado de los programas de estabilización y ajuste estructural que liberalizaron la economía, al abrir el comercio exterior y los mercados financieros, y redujeron las funciones del Estado en el desarrollo económico general y sectorial.

La forma actual de inserción de la agricultura (considerada en su sentido amplio, que comprende cultivos, ganadería y silvicultura, así como la transformación industrial primaria de los productos del campo) en la economía global se ha visto también condicionada por la conclusión de la Ronda Uruguay del GATT, y ha alcanzado su expresión más acabada con la inclusión de México en diferentes tratados de libre comercio, entre los cuales los más importantes son el TLCAN (Estados Unidos, Canadá y México) y el TLCUEM (Unión Europea y México).

Actualmente, como resultado de la rapidez con que se han desarrollado estos procesos, la agricultura mexicana atraviesa por una situación bastante crítica. Sucede que desde 1982 a la fecha: 1) cae el valor del PIB de la producción agropecuaria por habitante, disminuye drásticamente la producción per cápita de granos, declina la producción per cápita de carnes y lácteos, y disminuye severamente la producción maderable; 2) aumentan verticalmente las importaciones de alimentos, hasta superar el valor de las exportaciones petroleras; 3) se produce un agudo proceso de descapitalización de la agricultura, que se manifiesta en el estancamiento cuantitativo y en la obsolescencia del parque de maquinaria agrícola, así como en la reducción del consumo productivo de insumos agrícolas modernos; 4) se registra un estancamiento cuantitativo y un deterioro cualitativo de la infraestructura agrícola, especialmente en obras de riego, infraestructura de investigación y servicios de apoyo a la producción; 5) se reduce el empleo en el sector y caen los salarios y los ingresos de todos sus trabajadores, privados y sociales; 6) se presenta la creciente insolvencia de los agricultores, tanto empresariales como campesinos, aumentando verticalmente el problema de las carteras vencidas y los requerimientos de apalancamiento financiero; 7) se complica la problemática social por las crecientes dificultades de los agricultores campesinos y empresariales para mantenerse en la producción agropecuaria como opción de trabajo y de vida, y por el deterioro de los niveles de bienestar que experimentan.

Esta nueva problemática, que se combina con viejos problemas no superados del sector agropecuario, hace necesaria la revisión de los esquemas teóricos sugeridos para su interpretación y la sistematización de nuevos enfoques que se han ido generando. Asimismo, se vuelve prioritaria la formación de recursos humanos del más alto nivel académico, capaces de analizar con rigor científico

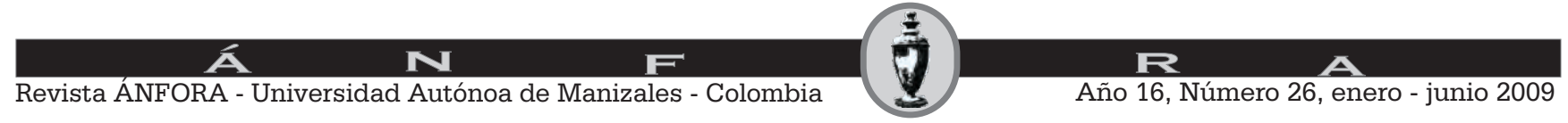


la nueva realidad agroindustrial, en su causalidad y manifestaciones específicas, a fin de generar propuestas alternativas que permitan a México retomar el camino del desarrollo agropecuario, aumentando el empleo y la oferta de alimentos, mejorando la productividad agregada, recapitalizando al campo y elevando los niveles de bienestar social.

Pocas experiencias teóricas y educativas parten de un diagnóstico nacional, del imperativo de formular una estrategia de desarrollo basada, en lo fundamental, en las condiciones y la problemática internas, y de las condiciones específicas de la actividad agropecuaria que son producto de nuestra historia. Hoy, sin desentenderse de la profunda interrelación que existe entre los países, y, en particular, sin subestimar el peso y la importancia del proceso de globalización y de los convenios internacionales que condicionan a la economía y al campo mexicano, el CIESTAAM a través del Doctorado en Problemas EconómicoAgroindustriales, ha formulado un proyecto teórico-académico que se fundamenta en la búsqueda de soluciones para las prioridades nacionales.

El análisis de la realidad económica, social y tecnológica que determina los resultados de la actividad productiva de los campesinos y empresarios agrícolas mexicanos evidencia que está conformada por dos grandes géneros de problemas:

1. Aquellos que caen en el ámbito de las decisiones individuales o grupales de los agentes económicos, merced a las cuales intentan hacer un uso más eficiente de sus recursos productivos y elevar sus niveles de ingreso y bienestar. Este nivel de decisiones muestra que está influenciado por una práctica histórico nacional que se resiste a la difusión, adopción y generalización de los elementos foráneos innovadores, sin embargo, es un ámbito en el cual las instituciones públicas académicas y gubernamentales, así como las organizaciones de los sectores social y privado pueden realizar acciones que, a un costo relativamente bajo, contribuyan a que las decisiones que se tomen sean las mejores. Se puede incidir de múltiples formas para introducir cambios para acrecentar la competitividad individual o generar alternativas en la mejora de las condiciones de vida, sin embargo, en muchas ocasiones es necesario realizar estudios específicos para demostrar su viabilidad.

2. Aquellos problemas que escapan de las decisiones individuales o grupales de los productores rurales, pero que determinan de manera relevante los resultados económicos individuales y agregados de la actividad agrícola. En este nivel se encuentran inscritas variables generales, como los coeficientes de intercambio, el precio del dinero, el tipo de cambio, la política de inversión o de crédito, las reglas del comercio, la generación de tecnología, los patrones de desarrollo y la difusión tecnológica, etc. Variables o decisiones en las que los sujetos individuales o grupales no pueden incidir o participar directamente.

Ambos géneros de problemas inciden, directa e indirectamente, en las posibilidades de desarrollo de la rama agropecuaria en un país de profundas raíces agrarias, tanto en los resultados agregados de la actividad agropecuaria 
nacional como en los microeconómicos de los productores campesinos o empresariales, determinando sus rendimientos, su solvencia económica, su ritmo de capitalización o descapitalización, su nivel de ingresos y, por ende, el grado de bienestar en que viven.

El uso más eficiente de los recursos, que conduzca a un más alto ingreso y a su correspondiente bienestar, implican, en este caso, una correspondencia entre las opciones que toman los productores, por una parte, y las acciones de las instituciones públicas, como administradoras del gasto público, de los servicios o de asistencia técnico-económica, o de inducción o capacitación organizativa, por la otra.

Ambas son una tarea que rebasa en el espectro más amplio a la administración pública y a los propios productores, vistos por separado. Encontrar, a través de la academia, el procedimiento para que la iniciativa en la formulación de políticas orientadas al sector agropecuario no considere solamente las variables macroeconómicas, sino que también incluya en sus premisas las condiciones específicas del cómo, es uno de los retos que nos proponemos enfrentar, ya que perder de vista la forma en que las grandes variables macroeconómicas inciden en el sector agropecuario y forestal es un error que cuesta muy caro al país en términos económicos y sociales. Pero un costo no menos alto deriva de la desatención de los problemas microeconómicos que pueden y deben encararse con eficacia.

El CIESTAAM, con el programa de Doctorado en Problemas EconómicoAgroindustriales impulsa su método y líneas de investigación y forma especialistas del más alto nivel académico, dotados de esta amplia visión macroeconómica, macrosocial, microeconómica y microsocial; es decir, trata de que los doctorandos, además de acceder a los conocimientos de carácter universal, aprendan una metodología de análisis de la realidad que les permita mantener una relación estrecha con los problemas reales para estudiar la pertinencia de estos conocimientos $\mathrm{y}$, en su caso, la perspectiva de su enriquecimiento, así como los límites de su aplicabilidad, a fin de que estén capacitados para analizar las grandes interrogantes del campo mexicano.

Se ha considerado necesario revisar los enfoques teóricos existentes sobre el desarrollo y los esquemas con que, en forma práctica, se ha diseñado la política agropecuaria y forestal de México, a fin de encontrar las mejores alternativas para asegurar que el país cuente con una sólida base de desarrollo económico y social en su sector agroalimentario (es decir, en las actividades agrícolas, ganaderas, forestales y agroindustriales). Asimismo, se ha hecho necesario confrontar las nuevas condiciones que presenta la inserción internacional del sector agroalimentario y las nuevas estrategias de política agrícola y rural, con las aspiraciones, potencialidades y condiciones específicas de las diversas regiones de México, cadenas agroalimentarias y grupos de productores, para encontrar un balance adecuado que permita mejorar las condiciones de vida de la población mayoritaria de productores, una garantía de permanencia rentable para los agentes de las cadenas agroalimentarias que atienden el mercado nacional y una capacidad del país para sostener la seguridad y autosuficiencia alimentaria.

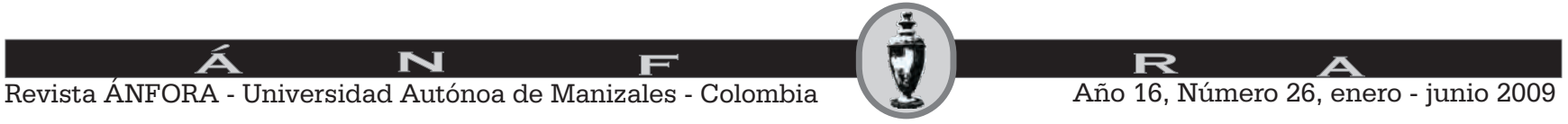




\section{VIABILIDAD DEL DOCTORADO}

La viabilidad del Doctorado se sustenta en la experiencia de investigación desarrollada por el CIESTAAM desde su fundación en agosto de 1990, la cual fue precedida por la experiencia de investigación desarrollada, desde diez años atrás, en programas interdepartamentales en los que participaron algunos de sus integrantes.

El Centro cuenta con una plantilla de investigadores nucleados a él como dependencia institucional, que han desarrollado un trabajo de investigación articulado en el programa general de investigación del Centro, que vincula orgánicamente la realización de estudios macroeconómicos, microeconómicos, ecológicos, sociológicos e históricos que se plasman en productos de investigación de carácter genérico, así como en productos de investigación por sistema producto, en los cuales el CIESTAAM tiene un amplio dominio y reconocimiento nacional e internacional. La prueba más evidente de estas aportaciones descansa en el reconocimiento de que la mayor parte de sus investigadores ha alcanzado mediante el ingreso al Sistema Nacional de Investigadores.

Este programa multidireccional de investigaciones le ha permitido al CIESTAAM retroalimentar metodologías entre las líneas de investigación y aportar análisis y respuestas científicas (al mismo tiempo que genéricas, congruentes con la realidad concreta) a grandes problemas nacionales, tales como la inclusión del sector agropecuario en el TLC de Norteamérica, el PROCAMPO, los problemas del crédito y aseguramiento, los impactos de la macrodevaluación, y hacer propuestas específicas a la problemática de un sinnúmero de sistemas agroindustriales.

Además, como el CIESTAAM ha construido vínculos con los más destacados investigadores de las instituciones académicas reconocidas en México, así como con prestigiados investigadores del extranjero, ha sido capaz de nuclear en su programa de Doctorado a investigadores del más alto nivel, que al interactuar con los doctorandos en cursos optativos, cursos elegibles y en los seminarios de investigación, generan espacios de crítica y reflexión sobre los principales problemas del agro mexicano.

Asimismo, el CIESTAAM ha sido sede de importantes congresos académicos, a los que han concurrido destacados investigadores de México y el extranjero. En estos congresos, las aportaciones han incluido tanto elementos que ofrecen alternativas para la corrección de políticas públicas, como estrategias específicas por producto de las que los sujetos del campo pueden tomar las opciones que más les convienen, desde la perspectiva económica y social. Todo ello se convierte así en un importante referente para los estudiantes del Doctorado.

En suma, la ejecución del Doctorado en Problemas Económico-Agroindustriales tiene su respaldo en los conocimientos generados por el CIESTAAM y en los recursos humanos del más alto nivel académico, así como en una amplia vinculación con instituciones nacionales e internacionales, y organizaciones de

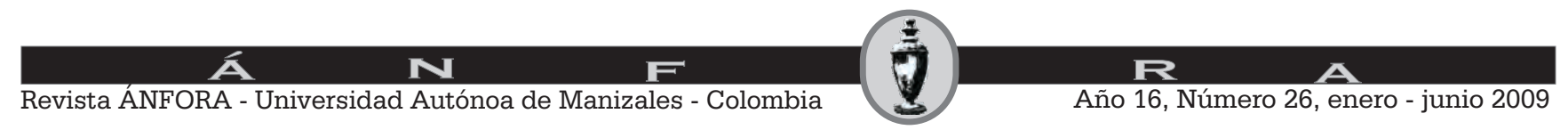


productores. Adicionalmente, su viabilidad se sostiene en la consolidación de la infraestructura física, el equipamiento con lo más avanzado en informática, la creación de un centro documental especializado y la diversificación de sus fuentes de financiamiento por medio del concurso ante CONACYT y la realización de trabajos de consultoría.

\section{OBJETIVOS DEL DOCTORADO}

Desde su origen, el Doctorado en Problemas Económico-Agroindustriales se propuso alcanzar el objetivo de preparar recursos humanos en el campo de los problemas económico agroindustriales contemporáneos, con una sólida formación que les posibilitara asimilar en forma crítica y constructiva los conocimientos concernientes al desarrollo agrícola y rural, y la vinculación agricultura-industria, para la resolución de problemas específicos, aplicando las metodologías adecuadas, recursos humanos que se actualicen mediante el estudio de los avances más recientes, que cotejen constantemente los resultados parciales de sus investigaciones con los principales actores de los sectores público, privado y social, de tal manera que los trabajos de investigación tengan un proceso continuo de retroalimentación y se obtenga así utilidad de lo estudiado, y que informen y difundan los resultados de sus investigaciones a través de artículos científicos, libros, seminarios y actividades de capacitación.

En otras palabras, se busca formar investigadores de alto nivel científico y técnico, con capacidad ejecutiva y una visión amplia que les permita incidir en la actual problemática del campo.

Además, los profesionistas así formados podrán comprender el papel del desarrollo agropecuario y agroindustrial en el modelo de acumulación de nuestro país, la articulación agricultura-industria y la conformación de cadenas agroindustriales y agroalimentarias.

Lo anterior se expresa formalmente en los objetivos del Doctorado:

\subsection{Objetivos Generales}

Generar conocimientos por medio de la realización de investigación original, evaluativa y propositiva sobre los problemas económicos, sociales y tecnológicos de la agricultura y la agroindustria mexicanas, con el fin de formar recursos humanos altamente capacitados mediante un proceso de enseñanzaaprendizaje sustentado en la investigación, que permita desarrollar al doctorando su potencial creativo e innovativo.

Para lograrlo, será necesario conjuntar la teoría del conocimiento y la experiencia en el medio rural, revisando y asumiendo críticamente las perspectivas de los productores y sus condiciones, para el diseño o el análisis de las políticas generales de desarrollo.

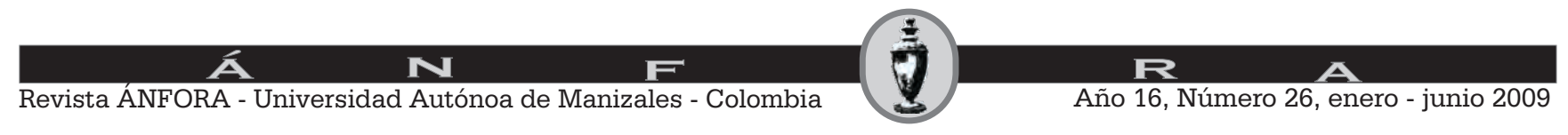




\subsection{Objetivos particulares}

- Formar especialistas de alto nivel académico en problemas económicoagroindustriales contemporáneos, capaces de elaborar propuestas de alternativas que permitan contribuir a resolver los problemas del sector agropecuario y agroindustrial, así como desarrollar estrategias de largo plazo.

- Consolidar un espacio académico de discusión y reflexión sobre la problemática del campo mexicano, en el cual participen los investigadores más destacados del país en la temática.

- Publicar los resultados en libros de editoriales de prestigio y en revistas de circulación nacional e internacional.

- Mantener una estrategia de difusión de los resultados de la investigación.

- Participar de diversas formas en la solución de los problemas que enfrenta el desarrollo del sector agroalimentario, a partir de los resultados de la investigación.

\section{PERFIL DE INGRESO AL DOCTORADO}

Actualmente, el Doctorado en Problemas Económico-Agroindustriales acepta anualmente un ingreso de no más de ocho estudiantes, con el fin de garantizarles buenas condiciones de trabajo. La estrategia para su selección se ha perfeccionado con la experiencia ganada. Los factores que se consideran son los siguientes:

1. Los alumnos del Doctorado deben destacarse por su actitud positiva para el trabajo individual y en equipo de investigación, su habilidad innovadora y creativa, capacidad de análisis y abstracción, así como por conocimientos en la temática y formación metodológica.

2. Para la admisión al estudio del Doctorado se requiere el grado académico de Maestro en Ciencias en un campo afín a la orientación del campo electo, como pueden ser economía agrícola, sociología rural, administración de empresas, etc., y tres años de experiencia profesional, demostrable en actividades de investigación.

3. El aspirante debe presentar una propuesta de investigación, cristalizada en un anteproyecto, acorde con las líneas de investigación del Doctorado.

4. El aspirante debe poseer un dominio de inglés y/o francés, que le permita leer textos en estos idiomas (450 puntos de TOEFL, comprobados en examen del Centro de Idiomas de la UACh, con fecha no mayor a 12 meses del examen de admisión). Manejar paquetes de cómputo como: procesador de texto, hoja de cálculo, graficador y elaborador de diapositivas; es deseable también que domine algún paquete de análisis estadístico, como SAS o SPSS.

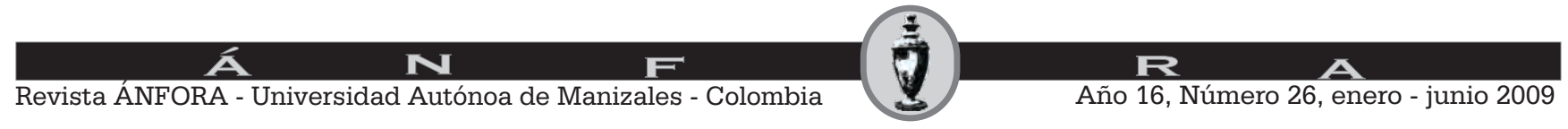


5. Adicional a lo anterior, y con la intención de propiciar la formación de investigadores jóvenes, con capacidad para sustituir a los grandes cuadros de intelectuales nacionales, sin demérito de la calidad, y con el objetivo de evitar que la mayor parte de los estudiantes del Doctorado sean profesores de la propia institución, es decir, lo que se conoce como "endogamia". No más del $25 \%$ de los solicitantes aceptados podrán ser profesores de la UACh.

6. No más del $25 \%$ de los solicitantes aceptados podrán tener más de 40 años.

7. Mínimo el 25\% de los estudiantes serán mujeres.

\section{PLAN DE ESTUDIOS}

\subsection{Objetivos del Plan de Estudios}

1. Ofrecer una base teórica actualizada sobre los problemas del desarrollo, las teorías económicas que sustentan las estrategias de política macroeconómica y sectorial, la estructura y funcionamiento de las cadenas agroalimentarias, y el desarrollo sustentable a fin de que los estudiantes sean capaces de interpretar la realidad actual del sector agroalimentario mexicano.

2. Proporcionar a los estudiantes un conjunto de herramientas del campo de la economía, que les permitan el manejo de la información necesaria para la comprensión de los fenómenos bajo estudio.

3. Generar las condiciones para que los estudiantes conozcan los distintos enfoques existentes sobre metodología de investigación y desarrollen habilidades prácticas para la realización de sus proyectos de tesis.

4. Proporcionar espacios de trabajo y reflexión colectiva que permitan un permanente contacto con los problemas del sector agroalimentario y una actualización, a fin de que sus trabajos de investigación mantengan un carácter propositivo y de aplicación práctica.

5. Propiciar el desarrollo de habilidades para el trabajo colectivo y la dirección de grupos de trabajo.

6.2. Metas del Plan de Estudios

1. En el primer año de ingreso, los alumnos estarán insertados en una línea de investigación y con un proyecto de investigación plenamente formulado en sus componentes esenciales.

2. Al final del segundo año de ingreso, los alumnos estarán en pleno desarrollo del trabajo de investigación de campo y realizando publicaciones conjuntas con sus asesores.

3. Al final del tercer año de ingreso, los alumnos serán capaces de presentar su examen predoctoral y de concluir el borrador de tesis, asimismo registrarán participación en eventos científicos y/o en actividades de capacitación.

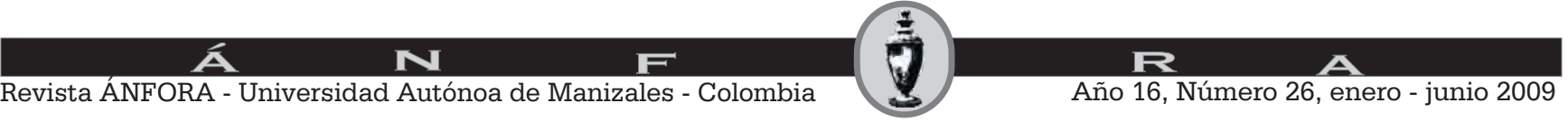


4. Durante el cuarto año, todo egresado deberá concluir con la presentación de su examen de grado.

\subsection{Estructura del Plan de Estudios}

A continuación se presenta el mapa curricular del Doctorado en Problemas Económico-Agroindustriales, como base para abordar su lógica interna.

Como se aprecia en el mapa curricular, el Plan de Estudios está organizado en cinco grupos de actividades académicas para cumplir con los objetivos propuestos: cursos obligatorios, seminarios de investigación, talleres de investigación, problemas especiales y actividades complementarias y ha sido diseñado con un alto grado de flexibilidad, buscando resolver con él las distintas circunstancias que se presentan en cuanto al perfil de los estudiantes, su trayectoria profesional y su nivel académico.

Las materias agricultura y desarrollo rural, macroeconomía, métodos cuantitativos I, política agrícola y agroindustrial, microeconomía y métodos cuantitativos II son cursos obligatorios. Con este tipo de cursos se pretende cumplir con los primeros dos objetivos del Plan de Estudios, que se refieren a ofrecer bases teóricas para comprender el desarrollo del sector agroalimentario y herramientas económicas que permitan el manejo de la información respectiva. La finalidad es proporcionar las bases al estudiante para preparar su tema de investigación y decidir el enfoque que habrá de darle al problema de estudio seleccionado.

Los seminarios de investigación, cursos obligatorios, representan el espacio en el que se ofrece conocimiento sobre el método de investigación y se apoya la elaboración de los proyectos particulares de tesis de cada doctorando, se someten a discusión colectiva sus avances y se permite el intercambio de experiencias y puntos de vista con investigadores de otras instituciones, de la UACh y del mismo CIESTAAM. Estos seminarios se programan en todos los semestres del Doctorado, considerando que la elaboración de la tesis es una de las principales prioridades.

Con los seminarios se cumplen parcialmente los objetivos tercero y cuarto del Plan de Estudios. La forma práctica en que se desarrollan, los objetivos particulares, contenido y formas de evaluación de cada seminario puede apreciarse en el Anexo 1.

Los talleres de investigación son cursos obligatorios, y cumplen la función de ser espacios de creación de habilidades prácticas para realizar investigación, y de promoción de la discusión y del trabajo colectivo e interdisciplinario entre estudiantes y profesores. En estos talleres se transmite la experiencia que el CIESTAAM ha desarrollado para abordar el estudio de problemas particulares y generar resultados que se ofrecen al país. Por tal razón, su contenido es variable, en función de la coyuntura que se presenta de un año a otro. En el Anexo 1 se presenta el programa analítico, objetivos y forma de evaluación respectivos. La ubicación de esta materia en verano sólo es indicativa de que es en el período vacacional cuando se realiza el trabajo de campo.

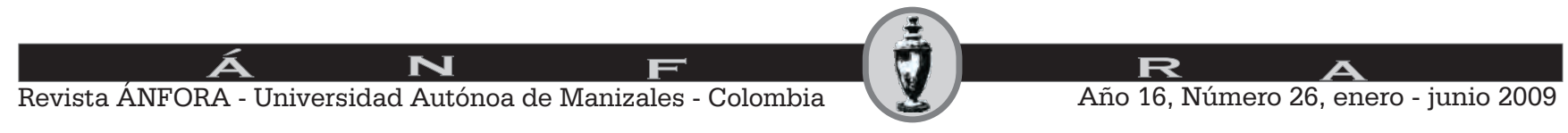


Los problemas especiales son un espacio diseñado con valor curricular para ofrecer a los estudiantes la oportunidad de que lleven cursos elegibles o cursos optativos. Los primeros son programados por el estudiante en acuerdo con su comité asesor y tienen la finalidad de fortalecer áreas específicas que se consideran útiles para su trabajo de investigación. El CIESTAAM está abierto para los cursos elegibles puedan tomarse en otra institución nacional o incluso en el extranjero. Por su parte, los cursos optativos son ofrecidos por el mismo Centro responsable de este Doctorado, ya sea mediante su propio personal docente, o acudiendo a la colaboración de profesores de otras instituciones nacionales y del extranjero. En este caso, el Comité del Posgrado está muy atento a las necesidades de las temáticas que cada año trabajan los estudiantes y a la coyuntura que se presenta a nivel nacional e internacional, para identificar las temáticas de cursos más convenientes. En el Anexo 1 se precisan las ideas anteriores y se ejemplifica el tipo de cursos que se han ofrecido.

Cuadro . Plan de Estudios del Doctorado

\begin{tabular}{|c|c|c|}
\hline Año y periodo & Curso & Créditos \\
\hline $\begin{array}{l}\text { Primer año, } \\
\text { Otoño } \\
\text { Subtotal }\end{array}$ & $\begin{array}{l}\text { Agricultura y Desarrollo Rural } \\
\text { Seminario de Investigación I } \\
\text { Seminario General de Investigación* } \\
\text { Inglés }\end{array}$ & $\begin{array}{l}3 \\
4 \\
\text { S.c } \\
7\end{array}$ \\
\hline $\begin{array}{l}\text { Primer año, } \\
\text { Primavera } \\
\text { Subtotal }\end{array}$ & $\begin{array}{l}\text { Políticas Agrícolas y Agroindustri ales } \\
\text { Seminario de Investigación II } \\
\text { Seminario General de Investigación* } \\
\text { Problema especial I (Curso elegible/optativo) } \\
\text { Inglés (Examen TOEFL, } 420 \text { puntos) }\end{array}$ & $\begin{array}{l}3 \\
4 \\
3 \\
\text { s.c. } \\
10\end{array}$ \\
\hline $\begin{array}{l}\text { Primer año, } \\
\text { Verano } \\
\text { Subtotal }\end{array}$ & Taller de Investigación I & $\begin{array}{l}4 \\
4\end{array}$ \\
\hline $\begin{array}{l}\text { Segundo año, Otoño } \\
\text { Subtotal }\end{array}$ & $\begin{array}{l}\text { Seminario de Investigación III } \\
\text { Seminario General de Investigación* } \\
\text { Problema especial II (Curso elegible/optativo) } \\
\text { Inglés } \\
\text { Actividades complementarias }\end{array}$ & $\begin{array}{l}4 \\
3 \\
\text { s.c. } \\
\text { s.c. } \\
7\end{array}$ \\
\hline $\begin{array}{l}\text { Segundo año, } \\
\text { Primavera } \\
\text { Subtotal }\end{array}$ & $\begin{array}{l}\text { Seminario de Investigación IV } \\
\text { Seminario General de Investigación* } \\
\text { Inglés (Examen TOEFL, } 460 \text { puntos) } \\
\text { Actividades complementarias }\end{array}$ & $\begin{array}{l}4 \\
\text { s.c. } \\
\text { s.c. } \\
4\end{array}$ \\
\hline $\begin{array}{l}\text { Segundo año, } \\
\text { Verano } \\
\text { Subtotal }\end{array}$ & Taller de Investigación II & $\begin{array}{l}4 \\
4\end{array}$ \\
\hline
\end{tabular}




\begin{tabular}{|l|l|l|}
\hline $\begin{array}{l}\text { Tercer año, } \\
\text { Otoño }\end{array}$ & $\begin{array}{l}\text { Seminario de Investigación V } \\
\text { Seminario General de Investigación* } \\
\text { Predoctoral } \\
\text { Investigación III } \\
\text { Inglés }\end{array}$ & 4 \\
Subtotal & & 3 \\
\hline $\begin{array}{l}\text { Tercer año, } \\
\text { Primavera }\end{array}$ & $\begin{array}{l}\text { Seminario de Investigación VI } \\
\text { Seminario General de Investigación* }\end{array}$ \\
Subtotal & Idiomas (Examen Toefl 500 puntos) & $\mathbf{1 0}$ \\
\hline Tercer año & & 4 \\
\hline Total Opción 1 & Presentación de la tesis de grado & $\mathbf{4}$ \\
\hline
\end{tabular}

s.c.: $\sin$ crédito

* El Seminario General de Investigación es una de las principales actividades académicas del Doctorado y del CIESTAAM, se realiza desde 1996 cada 15 días los días jueves. En el marco de este seminario se presentan los avances de tesis al final de cada semestre. Forma parte de los Seminarios de Investigación de cada semestre y se califica como tal. La asistencia a este seminario es obligatoria para maestros y alumnos, y ningún alumno puede tener más de dos faltas para acreditar el Seminario de Investigación del semestre respectivo.

Las actividades complementarias, aunque no tienen valor curricular, se programan en el Plan de Estudios porque la experiencia ha demostrado que los estudiantes pueden fortalecer su formación y su trabajo de tesis participando en proyectos de los investigadores del CIESTAAM, acudiendo a eventos e incluso realizando estancias en otros centros de investigación del país o del extranjero.

La coordinación del Doctorado está abierta a la realización de estancias semestrales o hasta por un período de un año en universidades extranjeras, con las cuales se mantiene una colaboración mediante convenio, en función de las necesidades particulares de los estudiantes. Lo anterior puede ser realizado a partir del segundo año y se garantiza la revalidación de los créditos en las materias correspondientes.

Con la exposición anterior se deja claro que el alumno recibe una fuerte formación orientada a la investigación, ya que las actividades académicas de este posgrado se definen a partir de conceptuar que su objeto de estudio abarca no solo los problemas económico-agroindustriales en sí, sino además: a) el condicionamiento social e institucional del doctorando; b) su campo de especialización y área particular de interés y c) la actividad o proceso de investigación por sí misma.

Al considerar lo anterior en conjunto con el objetivo general del Doctorado, se han definido las actividades académicas, a las cuales también se les ha asignado un orden de prioridad, de acuerdo con la importancia que tienen para el participante en este programa doctoral:

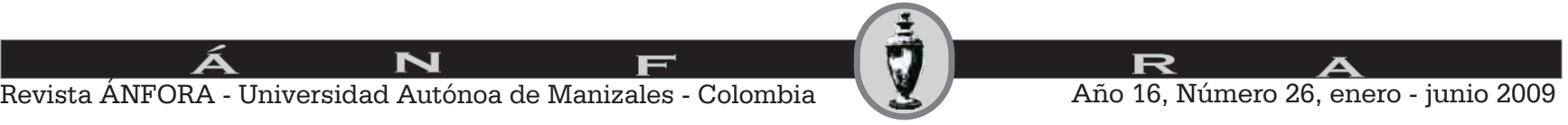


1. Cursos teórico-metodológicos

2. Investigación para tesis

3. Seminarios de investigación

4. Talleres de Investigación

5. Problemas especiales

6. Actividades complementarias

\author{
Prioridad uno \\ Prioridad uno \\ Prioridad uno \\ Prioridad uno \\ Prioridad dos \\ Prioridad tres
}

Bajo este esquema de actividades académicas, en el Doctorado se retoman las mejores experiencias histórico-internacionales y se recalca en el trabajo colectivo, al hacer que el estudiante forme parte de equipos de investigación interdisciplinaria y se involucre en la dinámica y mística del trabajo de dichos grupos de investigación. Actualmente varios estudiantes se encuentran integrados a los equipos de investigación de los tres programas que conforman al CIESTAAM.

El desarrollo de todas estas formas de trabajo durante el Doctorado también contribuyen a capacitar y a formar al estudiante, a través de un proceso de autoaprendizaje que lo obliga a desarrollar sus capacidades individuales, además de que se fortalece el proceso formativo mediante la dirección de los tutores y con la participación del doctorando en los espacios de discusión y reflexión de los seminarios y cursos-taller de investigación.

\section{MECANISMOS PARA EL SEGUIMIENTO DE AVANCES DE LOS DOCTORANDOS}

El Doctorado del CIESTAAM cuenta con los siguientes mecanismos para el seguimiento de la trayectoria de los estudiantes: El conjunto de cursos obligatorios, el seminario de investigación semestral, un seminario general quincenal, un comité de asesores por alumno y el Comité del Posgrado, integrado por los profesores de base y tiempo completo.

En los cursos los profesores conocen a fondo el potencial de cada estudiante y establecen formas de relación entre sus materias y las necesidades de los distintos temas de investigación, por medio de tareas y ejercicios prácticos.

Cada seminario se orienta por la definición de las metas y objetivos a lograr en un semestre y se enriquece con la participación de varios profesores, además del coordinador responsable. En él se solicita un programa semestral de actividades a cada estudiante en común acuerdo con sus asesores y se busca la interacción entre estudiantes y de éstos con los profesores, a partir de la exposición y presentación de avances escritos, que son discutidos a lo largo de cada semestre.

En la práctica, esta forma de ejecución de los seminarios permite un seguimiento individualizado, y detectar los problemas de cada estudiante. Como resultado, se han desprendido actividades específicas, por ejemplo, la asignación de asesores adicionales al grupo formal de tutores, con el fin de realizar un seguimiento puntual de los avances de investigación.

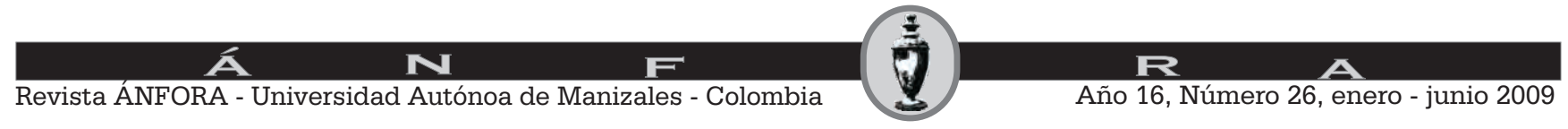


En el seminario general se ha buscado crear un foro de análisis de la problemática agroalimentaria de México, con el concurso de investigadores del CIESTAAM, de la UACh y de otras instituciones nacionales e internacionales. Al mismo tiempo que se amplía el panorama sobre lo que ocurre en el ámbito mundial y nacional, las diversas exposiciones permiten identificar distintos enfoques para la investigación, y con ello, aportaciones metodológicas y teóricas.

Adicionalmente, al final del semestre, en el seminario general se programa una exposición de avances de cada estudiante, exámenes predoctorales y exámenes de grado. Se solicita la entrega de los resultados escritos de cada trabajo, previo a su presentación oral, con el fin de asignar un comentarista interno o de otra institución. Este es otro de los mecanismos que se utiliza para dar seguimiento al trabajo de investigación de cada estudiante, lo cual junto con los comentarios y sugerencias de alumnos y profesores asistentes a este seminario, y las recomendaciones de los comentaristas externos, permite mejorar la calidad de las investigaciones del doctorando.

En la designación que hace el Comité de Posgrado de cada comité particular de asesores, que se integra por el director de tesis y dos asesores, se procura que exista correspondencia entre las líneas de investigación, la experiencia particular de cada maestro y el tema de interés del estudiante. Aunque cada comité particular de asesores trabaja en forma libre e independiente con el alumno, por medio de su participación en las distintas actividades del Doctorado se posibilita el seguimiento de los avances de los tesistas.

Finalmente, el Comité de Posgrado del Doctorado tiene establecida una reunión mensual para el seguimiento de las actividades docentes y la solución de los asuntos operativos y administrativos. Como parte de sus actividades, en este órgano académico-administrativo se da seguimiento a la trayectoria de cada doctorando. En realidad es aquí donde se intercambia la información que se obtiene en los cursos, seminarios y asesorías de tesis, en relación con los avances individuales de los doctorandos. Como consecuencia, en el Comité de Posgrado se deciden actividades específicas de apoyo o determina recomendar la suspención temporal de los estudios al o los alumnos que se considera no han logrado la calidad o los avances suficientes en su investigación.

En síntesis, los mecanismos más importantes de seguimiento académico a los estudiantes son los seminarios semestrales y el seminario general. El Comité de Posgrado es la instancia que formaliza las recomendaciones de actividades adicionales o alternativas para cada alumno.

Entre las principales actividades específicas que se han establecido para apoyar la mejora de las investigaciones se puede destacar la discusión interactiva de cada tema, la incorporación de varios profesores en un seminario particular, la participación de comentaristas externos en cada trabajo de investigación y la asesoría personalizada, adicional a los grupos formales de asesores.

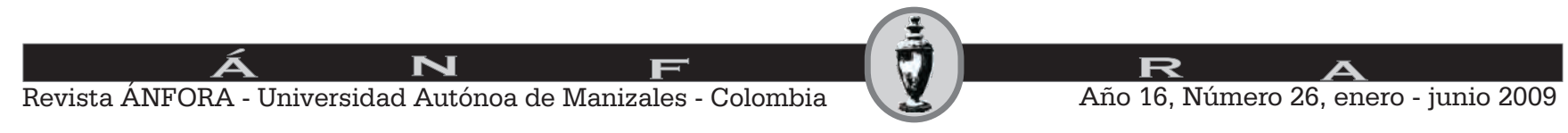




\section{LÍNEAS DE INVESTIGACIÓN}

El programa de Doctorado se sustenta en tres líneas de investigación que son: Estructura y dinámica de los sistemas agroindustriales; Análisis de políticas agrícolas y agroindustriales; y Desarrollo rural sustentable. Todos los proyectos de tesis de los doctorandos deberán estar insertos en alguna de ellas.

\section{Estructura y dinámica de los sistemas agroindustriales}

Sus objetivos fundamentales son:

- Impulsar actividades académicas de tipo colectivo, multidisciplinario, interdepartamental e interinstitucional para formular propuestas integrales a la problemática de los sistemas agroindustriales.

- Identificar la problemática presente en los sistemas agroindustriales del país, considerando prioritario el interés de los grupos sociales mayoritarios, para elaborar alternativas de solución acorde al contexto socioeconómico y de desarrollo en estos sistemas.

\section{Análisis de políticas agrícolas y agroindustriales}

Sus objetivos son:

- Analizar las políticas agrícolas y agroindustriales implementadas en el contexto nacional e internacional, así como identificar sus repercusiones a fin de difundir puntos de vista analíticos.

- Identificar los problemas en la aplicación de políticas agrícolas y agroindustriales en México, y generar planteamientos y alternativas de desarrollo ante los grandes problemas que enfrenta el país.

\section{Desarrollo rural sustentable}

Sus objetivos son:

- Realizar estudios que integren la dimensión ecológica, social y económica para promover el desarrollo rural.

- Analizar el desarrollo rural en el contexto económico, social y ambiental para generar diferentes alternativas. También, diseñar políticas públicas que permitan mejorar las condiciones de pobreza de la población rural y acercarla a una mayor equidad social con crecimiento económico, en una perspectiva comunitaria, regional y nacional.

\section{PERFIL DE EGRESO DEL DOCTORADO}

Los egresados del Doctorado en Problemas Económico-Agroindustriales contarán con un conjunto de conocimientos y habilidades que les permitirán entender y participar en la dinámica actual del sector agroalimentario, asumir sus retos fundamentales y ubicar con conocimiento de causa las oportunidades

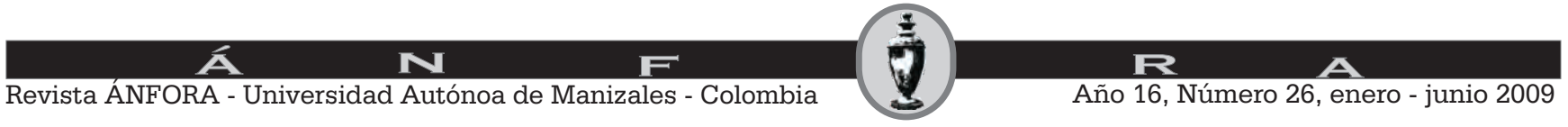


que como país deben ser aprovechadas en el actual contexto internacional. Con una visión crítica y una sólida formación, dispondrán de los instrumentos necesarios para brindar consultoría y asesoría, incorporarse en equipos de trabajo y dar orientaciones sobre alternativas de política agroalimentaria, diseño y ejecución de planes y programas particulares, y decisiones sobre proyectos específicos de carácter productivo. De esa forma, estarán capacitados para participar en los sectores social, privado y público: organizaciones de productores, empresas en lo individual y dependencias gubernamentales (SAGARPA, SEDESOL, Secretaría del Medio Ambiente, etc.). En particular, serán capaces de:

- Realizar investigación propia y original.Crear y dirigir grupos de investigación

- Plantear alternativas viables a la problemática del sector rural.

- Participar en la formación de recursos humanos en docencia e investigación.

- Difundir los resultados de investigación.

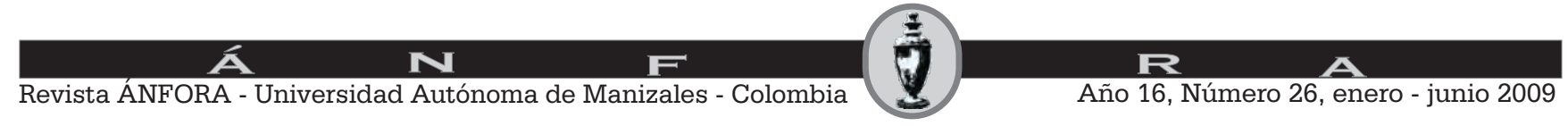

